\title{
Syndromic Testing by BioFire FilmArray The Right test, the First time.
}

Let us talk today on a new-age syndromic testing platform. A multiplex polymerase chain reaction (PCR) guides you in the right direction in complex diagnostic situations. The results are available in an Hour! The syndromic approach helps in ruling in and ruling out of multiple targets like viruses, bacteria, fungi, parasites at one go.

Currently, hospitals and labs take days, weeks and, sometimes, months, to diagnose the organism that is causing the disease since there are countless possibilities of organisms that are making patient sick. Diagnosing these infections in timely manner was challenging to the lab since there was no technology available so far. This delay in diagnosis could cause delay in appropriate treatment, patient management and therefore increase in mortality, morbidity, length of stay, days on antibiotics and ultimately overall patient cost.

The BioFire ${ }^{\circledR}$ FilmArray ${ }^{\oplus}$ system is a comprehensive one-hour syndromic test. Each test panel targets a comprehensive grouping of pathogens that could be causing a specific syndrome and the system easily integrates into clinical laboratories, allowing for relevant molecular testing around the clock without any prerequisites, infrastructural changes or difficult-to-maintain workflows unlike other conventional molecular systems. It streamlines laboratory workflow and maximizes productivity in an easy, fast, and comprehensive way.

It is easy to perform at lab level too, the technicians simply prepare the test in two minutes without precise measuring or pipetting. They load the pouch onto the system feed in the details using barcode and that's it. The sample is automatically analyzed and results are reported in an easy-to-read format.

This system has helped the lab attain meaningful answers fast and therefore been useful for clinicians, the hospital and patients

... It's a HIGH clinical value test used in:

- Urgent situations - e.g. in meningitis/ encephalitis, pneumonia condition

- Difficult diagnostic situations - viruses are not easily isolated or detected

- For critical patients - neonates, infants, elderly people, immunocompromised, oncology cases

Using this test will have high impact on patient management and healthcare costs:

- It guides to early therapeutic adjustment - viral vs bacterial vs fungal.

- It's a tool for better and safer patient isolation - e.g. in flu season. This can identify influenza-A/H1N1 in one hour. Also, it will provide information on influenza $A$ and $B$ and influenza-A/H3.

- Improved patient management (reduction in nosocomial infections, shorter time to right therapy, length of stay...)

- A significant impact on healthcare costs (reduced length of stay, reduced use of antimicrobial, reduced rate of nosocomial infection, reduced number of downstream healthcare procedures)

- Unlike conventional molecular tests where batching of samples is a standard practice, with FilmArray, we no longer batch our samples. One sample -1 test -1 hour. No overheads. It's a true $\mathbf{2 4 x 7}$ test. No risk of contamination as it's a completely closed system. Moreover, it does not need trained personnel for performing the test, anyone who is free for 2 mins can set the test and load away. No worries for cross-contamination from other tests or disturbing workflows. The USFDA and CE-IVD certification also puts us in a very comfortable position when reporting the results.

Hence, using the system has increased our productivity and efficiency with $100 \%$ confidence in the molecular front.

BioFire has so far launched 5 syndromic panels on the platform:

\begin{tabular}{|l|l|l|l|}
\hline $\begin{array}{l}\text { Pneumonia Plus } \\
\text { Panel }\end{array}$ & 34 Targets & Bacteria, Viruses, Antibacterial resistance genes & $\begin{array}{l}\text { Sputum, ET, BAL, mini-Bal, in sterile } \\
\text { Container }\end{array}$ \\
\hline ME Panel & 14 Targets & Bacteria, Viruses and Fungi & CSF in sterile container \\
\hline GI Panel & 22 Targets & Bacteria, Viruses and Parasites & Loose stool in Cary-Blair media \\
\hline BCID Panel & 27 Targets & Bacteria and Antibacterial resistance genes & Positive blood culture \\
\hline RP2Plus Panel & 22 Targets & Bacteria and Viruses & $\begin{array}{l}\text { Nasopharyngeal swab in viral transport } \\
\text { medium (VTM) }\end{array}$ \\
\hline
\end{tabular}

Whether deciding on empiric therapy for a septic patient or determining exactly which respiratory pathogen is making a young child sick, FilmArray can return answers fast. Physicians attain answers sooner and laboratories maximize productivity and reduce costs.

In India, BioFire FilmArray panels are available, courtesy SRL Labs, the local partners who would ensure patient service \& logistics. The pathogen report comes in soft format on the clinician's phone. 